\title{
A Novel High Linearity and Low Power Folded CMOS LNA for UWB Receivers
}

\author{
Xin Zhang ${ }^{1, \mathbb{I}}$, Chunhua Wang ${ }^{1, *}$, Yichuang Sun ${ }^{2, \dagger}$, Haijun Peng ${ }^{1,+}$ \\ ${ }^{1}$ College of Computer Science and Electronic Engineering, \\ Hunan University, Changsha 410082, P.R.China \\ ${ }^{2}$ School of Engineering and Technology, University of Hertfordshire, \\ Hatfield Herts AL10 9AB, UK \\ Il zhangxin2302@hnu.edu.cn \\ $\triangle$,*wch1227164@ hnu.edu.cn \\ †.sun@herts.ac.uk \\ thaijunpeng@hnu.edu.cn \\ Received (24 February 2017) \\ Revised (13 June 2017) \\ Accepted (16 June 2017)
}

\begin{abstract}
This paper presents a high linearity and low power Low-Noise Amplifier (LNA) for Ultra-Wideband (UWB) receivers based on CHRT $0.18 \mu \mathrm{m}$ CMOS technology. In this work, the folded topology is adopted in order to reduce the supply voltage and power consumption. Moreover, a band-pass LC filter is embedded in the folded-cascode circuit to extend bandwidth. The transconductance nonlinearity has a great impact on the whole LNA linearity performance under a low supply voltage. A post-distortion (PD) technique employing an auxiliary transistor is applied in the transconductance stage to improve the linearity. The post-layout simulation results indicate that the proposed LNA achieves a maximum power gain of $12.8 \mathrm{~dB}$. The input and output reflection coefficients both are lower than $-10.0 \mathrm{~dB}$ over $2.5 \sim 11.5 \mathrm{GHz}$. The input third-order intercept point (IIP3) is $5.6 \mathrm{dBm}$ at $8 \mathrm{GHz}$ and the noise figure (NF) is lower than $4.0 \mathrm{~dB}$. The LNA consumes $5.4 \mathrm{~mW}$ power under a $1 \mathrm{~V}$ supply voltage.
\end{abstract}

Keywords: CMOS integrated circuits; UWB; LNA; post-distortion; high linearity; third-order distortion.

\section{Introduction}

Due to the rapid growth of wireless communications technologies, many wireless communication standards, such as Global System for Mobile Communications (GSM), Wideband Code Division Multiple Access (WCDMA), Long Term Evolution (LTE), Bluetooth and $802.11 \mathrm{a} / \mathrm{b} / \mathrm{g} / \mathrm{n}$, have been developed. To accommodate such broad range of services, multi-standard broadband radio receivers have drawn great attention. The LowNoise Amplifier (LNA) is usually the first on-chip active stage after antenna in almost all 
radio frequency (RF) and microwave receivers. As a critical module, LNAs in such receivers need to work for different standards and applications. An Ultra-Wideband (UWB) LNA which can be shared among different standards is preferred to save cost and reduce complexity. Such a LNA must provide good impedance matching, high linearity, low NF, and adequate gain across multi-GHz frequency band. ${ }^{1 .}$ For applications in mobile communication systems, low-voltage, low power and stability requirements are also important for LNA design. Stability is an important indicator to ensure the designed amplifier working properly. In terms of the reflection coefficient, the designed circuit system is stable only the modular of the reflection coefficient is less than one. If the value more than one, it makes reflected voltage larger than incident voltage, the whole system will oscillate because of positive feedback. ${ }^{2}$.

Up to now, several topologies have been proposed for UWB LNAs. The distributed amplifier (DA), ${ }^{3}, 4$. which absorbs the parasitic capacitance of the input transistor as part of the transmission line, can provide good operating performance over a wide range of frequency. However, the large area makes it less attractive to low cost applications and its relatively high power consumption is a limit to ultra-low power design. The shuntfeedback amplifier, ${ }^{5}$,6. which is a well-known topology used in wideband amplifiers, can provide broadband matching and flat gain over the entire bandwidth. Nevertheless, it is difficult to satisfy gain and noise performance simultaneously. The passive filtering configuration $^{7}$ is a good solution to extend bandwidth of amplifier. Inserting the Chebyshev filter in the input matching network can provide good wideband matching and high power gain. ${ }^{8}$ However, the NF is degraded by the insertion loss of the filter. Meanwhile, the large number of passive components will occupy a considerable chip area which will increase cost and size. The common-gate (CG) amplifier ${ }^{9}$. is popular in broadband LNA design for its excellent performance of wideband input matching and high linearity.

The linearity is of great importance for the UWB LNA design. It should be high enough to suppress interference and maintain high sensitivity. In a multi-standard wideband receiver, a wide range of out-band/in-band interferers may corrupt the signal, resulting in severe blocking, cross-modulation and inter-modulation, which pose a huge challenge to the linearity of the LNA. As the Complementary Metal Oxide Semiconductor (CMOS) technology scales down and supply voltage decreases, it is more difficult to obtain high linearity with a low supply voltage, since the nonlinear output conductance, short-channel and high-field mobility, which are ignored under a high supply voltage, become very important under low-voltage operation. The optimal biasing technique was proposed in Refs. 10. , in which the third-order derivative of DC transfer characteristic was adjusted to zero by regulating the biasing voltage $\mathrm{V}_{\mathrm{GS}}$. However, the bias voltage range for IIP3 peak is very narrow, making the linearity boosting very sensitive to the biasing voltage. The derivative superposition (DS) method is based on multiple-gated configuration, which adds the second/third-order derivatives from the main and auxiliary transistors to cancel distortion. It involves MOS transistors working in triode ${ }^{11 .}$ or weak inversion region, ${ }^{12 .}, 13$. therefore the DS method will find difficult 
to match the transistors working in different regions, resulting in a linearity improvement highly sensitive to process-voltage-temperature (PVT) variations. The post-distortion (PD) technique $^{14 .}, 15 . \quad$ is utilized to improve the linearity. Since all transistors are biased in the saturation region and some of them also avoid the input matching degradation, this technique has a robust increase of linearity.

In this paper, a high linearity, low power UWB LNA is introduced and a band-pass LC filter is used to extend bandwidth. The LNA adopts a CG structure of folded topology to provide input matching. In addition, to reduce the drain conductance nonlinearity, the PD technique is applied in the LC folded-cascode configuration. As a result, the UWB LNA has excellent performance even under an extremely low supply voltage.

\section{Theoretical Analysis of Linearity Improvement}

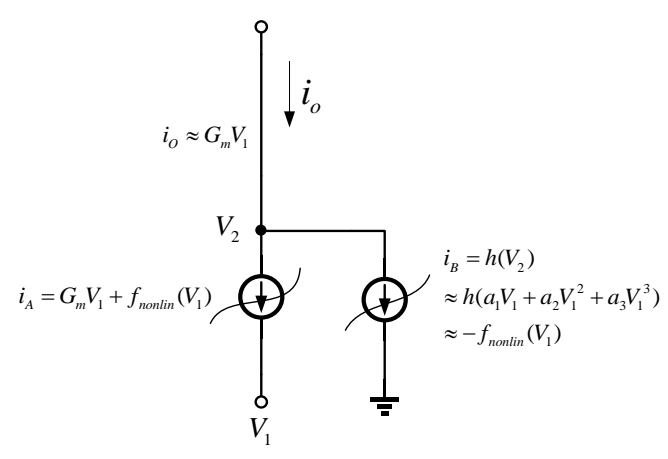

(a)

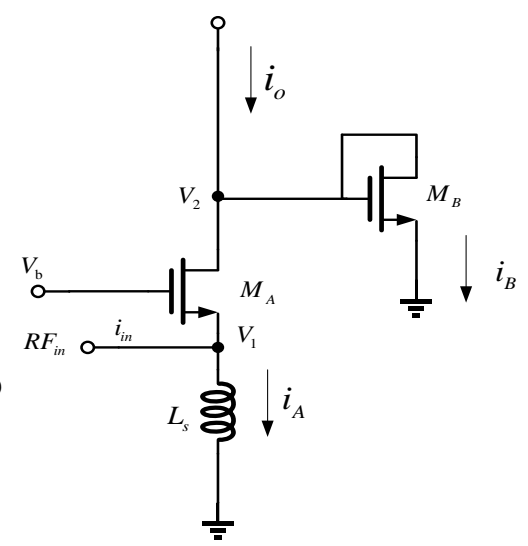

(b)

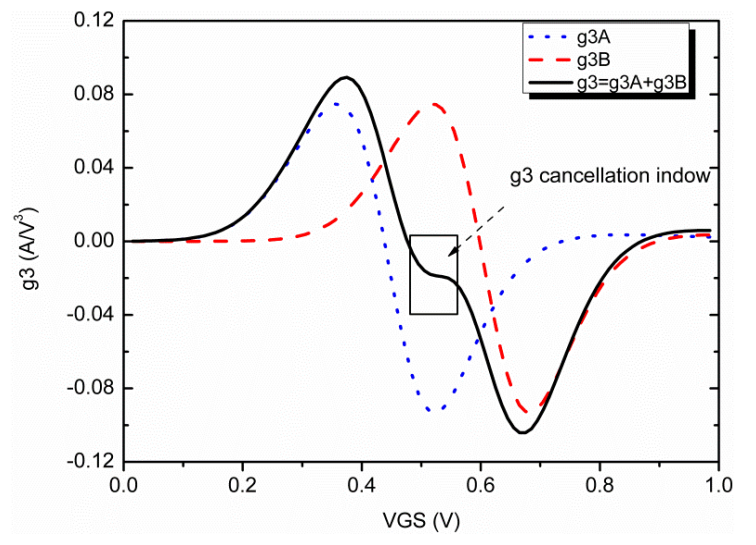

(c)

Fig. 1. The post-distortion technique: (a) Concept of the technique; (b) Circuit implementation; (c) Third-order power series coefficient of $i_{o}$ at DC. 
The drain current of a common-source (CS) Field Effect Transistor (FET) can be expressed in terms of gate-source voltage Vgs using the power series expansion:

$$
i_{d}=g_{1} v_{g s}+g_{2} v_{g s}^{2}+g_{3} v_{g s}^{3}
$$

where $g_{i}$ is the ith-order coefficient of transconductance. The nonlinear current generated in the CS FET is fully transferred to the next stage. If the drain node of the CS FET has an additional current path which selectively absorbs the third-order intermodulation distortion (IMD3) current component, then only the fundamental current component delivers to the next stage. A better linearity can thus be achieved. Here, the PD technique is utilized to design the proposed LNA circuit.

Figure 1(a) shows the PD technique which utilizes an auxiliary transistor $\left(M_{B}\right)$ 's nonlinearity to cancel that of the main device $\left(M_{A}\right)$. Both $M_{B}$ and $M_{A}$ operate in the saturation region with the same polarity. The transistor $M_{B}$ taps voltage $V_{2}$ and replicates the nonlinear drain current of the main transistor $M_{A}$, partially canceling secondorder/third-order distortion terms in Figs. 1(b)-1(c). The drain currents of $M_{A}$ and $M_{B}$ can be modeled as Eq. (2) and Eq. (3):

$$
\begin{gathered}
i_{A}=g_{1 A} V_{1}+\underbrace{g_{2 A} V_{1}^{2}+g_{3 A} V_{1}^{3}}_{f_{\text {nonlin }}\left(V_{1}\right)} \\
i_{B}=g_{1 B} V_{2}+g_{2 B} V_{2}^{2}+g_{3 B} V_{2}^{3}
\end{gathered}
$$

where $V_{1}$, and $V_{2}$ are the source and drain voltages of $M_{A}$, respectively. Suppose $V_{2}$ is related to $V_{l}$ by:

$$
V_{2}=-c_{1} V_{1}-c_{2} V_{1}^{2}-c_{3} V_{1}^{3}
$$

The currents have to satisfy the Kirchhoff's Current Law (KCL), yielding the output current $i_{o}$ :

$$
\begin{aligned}
i_{o} & =i_{A}+i_{B} \\
& =g_{p m 1} V_{1}+\underbrace{g_{p m 2} V_{1}^{2}}_{\begin{array}{c}
\text { second-order } \\
\text { distortion }
\end{array}}+\underbrace{g_{\text {pm } 3} V_{1}^{3}}_{\begin{array}{c}
\text { third-order } \\
\text { distortion }
\end{array}}
\end{aligned}
$$

where

$$
\begin{gathered}
\mathrm{g}_{p m 1}=g_{1 A}-c_{1} g_{1 B} \\
\mathrm{~g}_{p m 2}=g_{2 A}-c_{1}^{2} g_{2 B}-c_{2} g_{1 B} \\
\mathrm{~g}_{p m 3}=g_{3 A}-c_{1}^{3} g_{3 B}-g_{1 B} c_{3}-2 g_{2 B} c_{1} c_{2}
\end{gathered}
$$


The gate-to-source voltage $V_{g s}$ and the output drain current $i_{o}$ can be expressed in terms of $i_{\text {in }}$ using Volterra series expansion as below:

$$
\begin{aligned}
v_{g s}(j \omega)= & A_{1}(j \omega) \circ i_{i n}+A_{2}\left(j \omega_{1}, j \omega_{2}\right) \circ i_{i n}^{2} \\
& +A_{3}\left(j \omega_{1}, j \omega_{2}, j \omega_{3}\right) \circ i_{i n}^{3} \\
i_{o}(j \omega)= & B_{1}(j \omega) \circ i_{i n}+B_{2}\left(j \omega_{1}, j \omega_{2}\right) \circ i_{i n}^{2} \\
& +B_{3}\left(j \omega_{1}, j \omega_{2}, j \omega_{3}\right) \circ i_{i n}^{3}
\end{aligned}
$$

where "O" is the Volterra series operator" ${ }^{16 .}$ and for the coefficients $A_{1}, A_{2}, A_{3}, B_{1}, B_{2}$, and $B_{3}$, the reader can refer to the original paper. ${ }^{17} B_{n}$ can be calculated from Eqs. (5), (9), and (10) as follows:

$$
\begin{gathered}
B_{1}(j \omega)=g_{p m 1} A_{1}(j \omega) \\
B_{2}\left(j \omega_{1}, j \omega_{2}\right)=g_{p m 1} A_{2}\left(j \omega_{1}, j \omega_{2}\right)+g_{p m 2} A_{1}\left(j \omega_{1}\right) A_{1}\left(j \omega_{2}\right) \\
B_{3}\left(j \omega_{1}, j \omega_{2}, j \omega_{3}\right)= \\
g_{p m 1} A_{3}\left(j \omega_{1}, j \omega_{2}, j \omega_{3}\right)+2 g_{p m 2} \overline{A_{1}\left(j \omega_{1}\right) A_{2}\left(j \omega_{1}, j \omega_{2}\right)} \\
+g_{p m 3} A_{1}\left(j \omega_{1}\right) A_{1}\left(j \omega_{2}\right) A_{1}\left(j \omega_{3}\right)
\end{gathered}
$$

where

$$
\begin{gathered}
\overline{A_{1}\left(j \omega_{1}\right) A_{2}\left(j \omega_{1}, j \omega_{2}\right)}=\frac{1}{3}\left(A_{1}\left(j \omega_{1}\right) A_{2}\left(j \omega_{2}, j \omega_{3}\right)+A_{1}\left(j \omega_{2}\right) A_{2}\left(j \omega_{1}, j \omega_{3}\right)+\right. \\
\left.A_{1}\left(j \omega_{3}\right) A_{2}\left(j \omega_{1}, j \omega_{2}\right)\right)
\end{gathered}
$$

To calculate the two-tone third-order intermodulation distortion (AIP3), a two-tone excitement $\left(\omega_{a}, \omega_{b}\right)$ is applied to the input. The two-tone excitement and IIP3 equations are given by: ${ }^{8 ., 18 .}$

$$
\begin{array}{r}
\operatorname{AIP} 3\left(2 \omega_{b}-\omega_{a}\right)=\sqrt{\frac{4}{3}\left|\frac{B_{1}\left(j \omega_{b}\right)}{B_{3}\left(j \omega_{b}, j \omega_{b},-j \omega_{a}\right)}\right|} \\
\operatorname{IIP} 3\left(2 \omega_{b}-\omega_{a}\right)=\frac{A I P 3\left(2 \omega_{b}-\omega_{a}\right)^{2}}{8} \times R_{e}
\end{array}
$$

From Eqs. (11), (15), and (16), we can derive IIP3 by Volterra series analysis as:

$$
\begin{aligned}
I I P 3\left(2 \omega_{b}-\omega_{a}\right)= & \frac{1}{6 R_{e}} \cdot \frac{1}{\left\{Z_{s}(\omega)\right\} \cdot|H(\omega)| \cdot\left|A_{1}(\omega)\right|^{3} \cdot|\varepsilon(\Delta \omega, 2 \omega)|} \\
& \varepsilon(\Delta \omega, 2 \omega)=g_{p m 3}-g_{o B}
\end{aligned}
$$




$$
Z_{s}(\omega)=\frac{j \omega / C_{g s 1}}{-\omega^{2}+j \omega \frac{g_{p m 1}}{C_{g s 1}}+\frac{1}{L_{s} C_{g s 1}}}
$$

where

$$
g_{o B}=\frac{2}{3}\left(g_{p m 2}\right)^{2}\left\{\frac{2}{g_{p m 1}+A_{1}(\Delta \omega)}+\frac{1}{g_{p m 1}+A_{1}(2 \omega)}\right\}
$$

where

$$
\Delta(\omega)=\omega_{b}-\omega_{a}, \omega \approx \omega_{a} \approx \omega_{b}
$$

IIP3 is primarily affected by $\varepsilon(\Delta \omega, 2 \omega)$. Equations (17)-(20) implies that the linearity can indeed be improved by reducing $g_{p m 3}$. On the other hand, increasing $g_{p m 2}$ can increase $g_{o B}$ and this can further decrease $\varepsilon(\Delta \omega, 2 \omega)$. The post-distortion technique employs an additional folded diode to minimize $g_{p m 3}$. To obtain a good linearity, the coefficient of the third term in Eq. (5) should be close to zero, by adjusting the gate bias and size of MA and Мв.

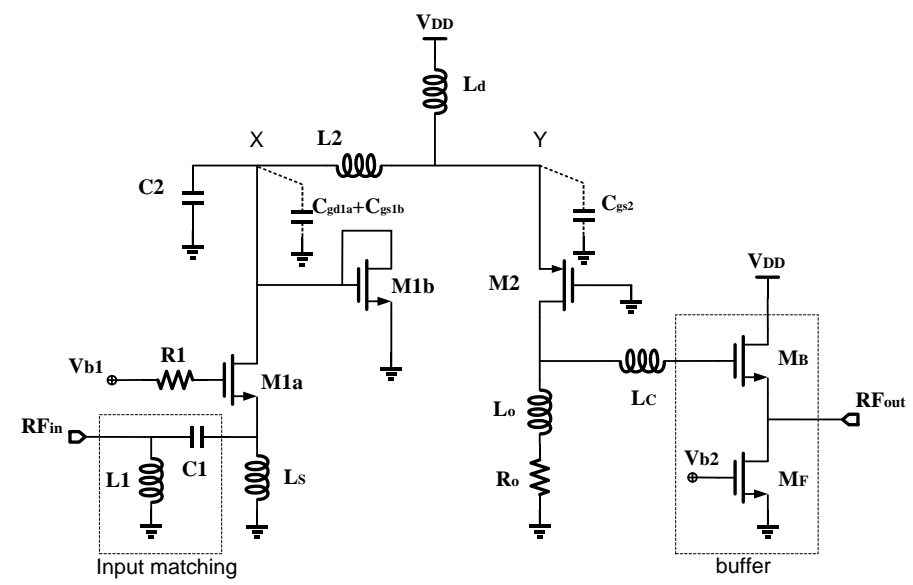

Fig. 2. The complete schematic of the proposed UWB LNA.

To achieve low power operation, the folded structure is a good choice since the transistors are placed in parallel between the supply DC voltage and GND and thus a large voltage can be avoided. Besides, good reverse isolation and stability in low power situation make this configuration more competitive especially in ultra-low power LNA design. Fig. 2 shows the complete circuit schematic of the proposed LNA which is composed of two gain stages. Taking advantage of the superior performance in broadband input matching, linearity, stability, and robustness to PVT variations, common-gate (M1a) structure is utilized as the input stage. Transistor M2 is the main 
transistor and M1b is the auxiliary transistor as a post-distortion circuit. All bodyterminals of the NMOS transistors are connected to GND in this LNA.

\section{Circuit Design and Analysis}

The design of LNA is based on the CHRT $0.18 \mu \mathrm{m}$ RF CMOS technology and all components are integrated. To take advantage of CG topology and folded structure as mentioned above, a folded CG broadband LNA is proposed. Fig. 2 shows a simplified circuit schematic of the folded cascode LNA where M1a and M2 represent the first and second gain stages, respectively. In the circuit implementation, the impedance matching at the input terminal is achieved by L1, C1 and Ls, while the Lc, Lo and Ro are adopted for output matching. As the inductor $\mathrm{Ld}$ resonates with capacitor $\mathrm{C} 2$ and inter-stage parasitic capacitor at the frequencies of interest, the bias current for M1a, M1b and M2 is provided without introducing excessive load to the gain stage. In conventional folded topology with RF choke inductor, Ld only ensures to deliver the RF signal current to the output terminal just within the narrow bandwidth of operation frequency. ${ }^{9}$ It is because the high impedance seen from the LC network is achieved around the resonant frequency of $\mathrm{Ld}$ and parasitic capacitor at the drain of M1a and source of M2/M1b. To improve the input wideband characteristic of CG M1a, the series inductor L2 is inserted at node X to broaden the bandwidth. The post-distortion technique is implemented at the first stage, reducing its influence on noise and IMD3.

\subsection{Wideband Matching}

\subsubsection{Input Matching}

Since the body-terminal of the NMOS transistor is connected to GND, the body effects and its parasitic capacitances are ignored in equation. For small signal analysis, the proposed LNA is treated as a two stage amplifier, and its simplified circuit model is illustrated in Fig. 3. From the equivalent circuit, the input impedance can be written as:

$$
Z_{\text {in }}=\frac{1}{\frac{1}{Z_{s}(\omega)}+\frac{1-g_{m \mid a} Z_{\text {ola }}(\omega)}{R_{o 1}+Z_{\text {ola }}(\omega)}} \approx \frac{1}{g_{p m 1}}=\frac{1}{g_{m 1 a}-c_{1} g_{m l b}}
$$

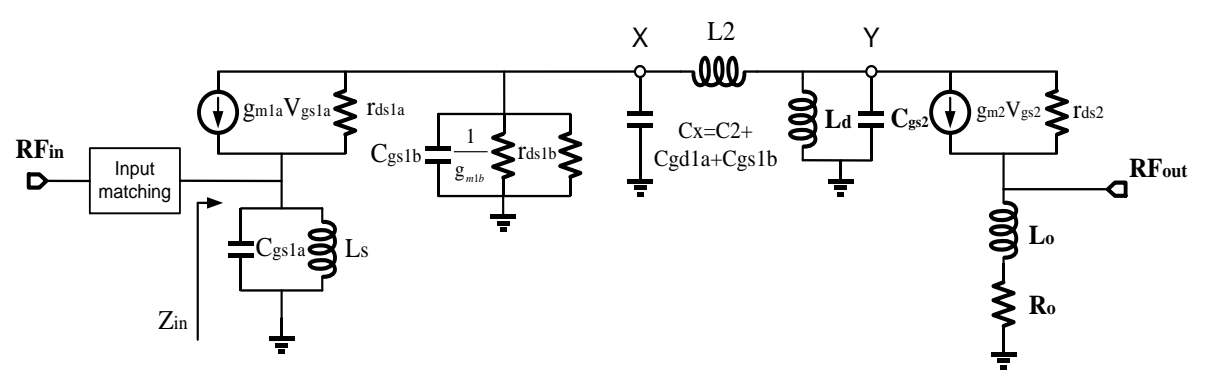

Fig. 3. Small signal model of the UWB LNA. 
The input impedance approximates $1 / g_{p m l}$ over the frequency band of interest. Due to the effect of other terms, $g_{m l a}$ should be set slightly greater than $32 \mathrm{mS}$. Through simulations, we have observed that the good input matching can be obtained when $g_{m l a}$ approaches to $34 \mathrm{mS}$.

\subsubsection{Bandwidth Extension Technique}

The LC $\pi$-network between $\mathrm{X}$ and $\mathrm{Y}$ can be redrawn as shown in Fig. 4(a). The $\pi$ network can be decomposed into two parts. The first part is constituted by L2 and $\mathrm{C}_{\mathrm{X}}$ as a lowpass filter (LPF) with a high cut-off frequency $\left(\omega_{\mathrm{L}}\right)$. The second part is formed by Ld and $\mathrm{C}_{\mathrm{gs} 2}$ as a narrow bandpass filter (BPF) with a lower peaking frequency $\left(\omega_{\mathrm{B}}\right)$. As seen from Fig. 4(a), the cascade of $2^{\text {nd }}$-order LPFs and $2^{\text {nd }}$-order BPFs, between node $X$ and $Y$ is a fourth-order doubly terminated bandpass filter.

(a)

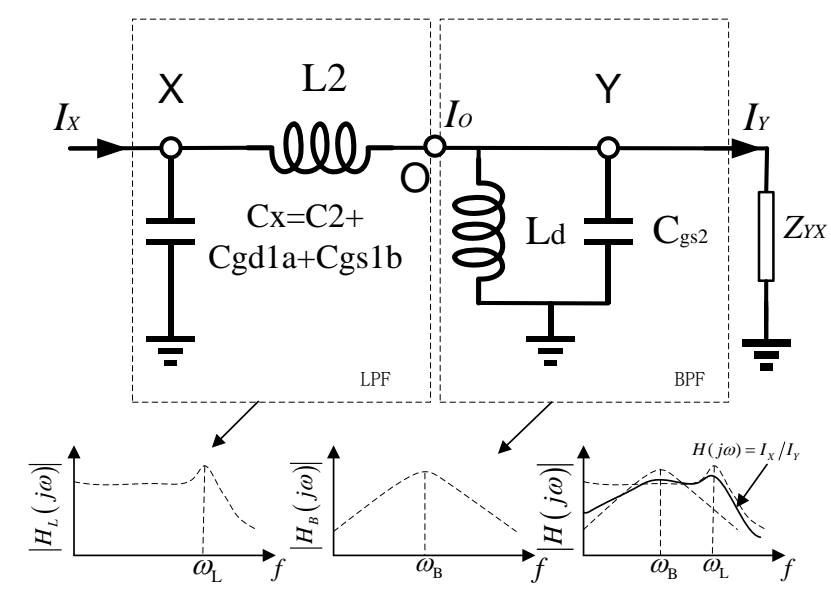

Fig. 4. The bandwidth extension circuit of the LC network between node X and Y.

From Fig. 4(b), we can write the transfer function $T_{I}(s)=I_{O} / I_{X}$ of $2^{\text {nd }}$-order LPFs and $T_{2}(s)=I_{Y} / I_{O}$ of $2^{\text {nd }}$-order BPFs equations as:

$$
\begin{gathered}
T_{1}(s)=\frac{I_{O}}{I_{X}}=\underbrace{\frac{k_{L} \omega_{L}^{2}}{s^{2}+\frac{\omega_{L}}{Q_{L}} s+\omega_{L}^{2}}}_{H_{L}}=\frac{\frac{1}{C_{X} L_{2}}}{s^{2}+\frac{Z_{O X}}{L_{2}} s+\frac{1}{C_{X} L_{2}}} \\
T_{2}(s)=\frac{I_{Y}}{I_{O}}=\underbrace{\frac{k_{B}\left(\omega_{B} / Q_{B}\right) s}{s^{2}+\frac{\omega_{B}}{Q_{B}} s+\omega_{B}^{2}}}_{H_{B}}=\frac{\frac{1}{Z_{Y X} C_{g s 2}} s}{s^{2}+\frac{1}{Z_{Y X} C_{g s 2}} s+\frac{1}{C_{g s 2} L_{d}}}
\end{gathered}
$$


where the $\mathrm{Z}_{\mathrm{Ox}}$ denotes impedance seen at $\mathrm{O}$ point into the BPF filter. And $Q_{B}$ and $Q_{L}$ are the pole quality factor of BPF and LPF, respectively. From Eqs. (23) and (24), the closedform formulas of $\omega_{B}, \omega_{L}, Q_{B}$, and $Q_{L}$ can be obtained as Eq. (25):

$$
\omega_{L}=\sqrt{\frac{1}{C_{X} L_{2}}}, Q_{L}=\frac{1}{Z_{O X}} \sqrt{\frac{L_{2}}{C_{X}}}, \omega_{B}=\sqrt{\frac{1}{C_{g s 2} L_{d}}}, Q_{B}=Z_{Y X} \sqrt{\frac{C_{g s 2}}{L_{d}}}
$$

From Fig. 4(a), it is clear that the circuit between node $\mathrm{X}$ to $\mathrm{Y}$ can be seen a cascade connection of $2^{\text {nd }}$-order LPF and BPF. The transfer function from node $\mathrm{X}$ to $\mathrm{Y}(H(j \omega)=$ $\left.\mathrm{I}_{\mathrm{Y}} / \mathrm{I}_{\mathrm{X}}\right)$ is derived as follows:

$$
\begin{aligned}
& H(s)=\frac{I_{Y}}{I_{X}}=H_{L} \bullet H_{B}=\underbrace{\frac{k_{L} \omega_{L}^{2}}{s^{2}+s \frac{\omega_{L}}{Q_{L}}+\omega_{L}^{2}}}_{L P F} \cdot \underbrace{\frac{k_{B}\left(\omega_{B} / Q_{B}\right) s}{s^{2}+s \frac{\omega_{B}}{Q_{B}}+\omega_{B}^{2}}}_{B P F} \\
& =\frac{\frac{L_{d}}{Z_{Y X}} s}{C_{X} C_{8 s 2} L_{2} L_{d} s^{4}+\left(\frac{1}{Z_{r X}} C_{X} L_{2} L_{d}+Z_{O X} C_{X} C_{8 s 2} L_{d}\right) s^{3}+\left(\frac{Z_{O X}}{Z_{r X}} C_{X} L_{d}+C_{X} L_{2}+C_{g s 2} L_{d}\right) s^{2}+\left(\frac{L_{d}}{Z_{Y X}}+Z_{O X} C_{X}\right) s+1}
\end{aligned}
$$

It is clear that Eq. (26) denotes transfer function of $4^{\text {th }}$-order BPF. Fig. 4(b) denotes the ADS simulation of frequency response of $2^{\text {nd }}$-order LPF, $2^{\text {nd }}$-order BPF and their cascade circuit. In the right-side figure of Fig. 4(b), two dotted lines denotes frequency responses of $2^{\text {nd }}-$ order LPF, $2^{\text {nd }}$-order BPF, and the solid line denote frequency responses of circuit between node $\mathrm{X}$ and $\mathrm{Y}$ in Fig. 4(a). It can be seen that frequency bandwidth of circuit between node $\mathrm{X}$ and $\mathrm{Y}$ became extension. Qualitatively, Input current signal $I_{X}$ below this cut-off frequency will be delivered to the output at node $\mathrm{Y}$ as $I_{Y}$. The resultant transfer function is a broadband $4^{\text {th }}$-order BPF characteristic as illustrated in Fig. 4(b). Then the wideband output matching is achieved. Taking M1b into account, the reactive resistance decreases and the capacitive reactance increases, due to M1b being placed in parallel with the load of M1a. Therefore we should slightly adjust the values of $\mathrm{C} 2$ and $\mathrm{L} 2$ to acquire a new wideband output matching. In this paper we use $\mathrm{C} 2=0.05 \mathrm{pF}$ and $\mathrm{L} 2$ $=6.5 \mathrm{nH}$.

\subsection{Gain Analysis}

Since the body-terminal of the NMOS transistor is connected to GND, the body effects and parasitic capacitances are not considered in equation. The voltage transfer function of the first stage can be expressed as:

$$
\begin{gathered}
A_{1}=\frac{v_{o 1}}{v_{i n}}=\frac{\left(g_{p m 1} r_{d s 1 a}+1\right)\left(Z_{d 1} / / \frac{1}{s C_{X}}\right)}{\left(R_{s} / / Z_{s 1}\right)\left(g_{p m 1} r_{d s 1 a}+1\right)+\left(Z_{d 1} / / \frac{1}{s C_{X}}\right)+r_{d s 1 a}} \\
Z_{d 1}=Z_{i n 2} / /\left(R_{d 1}+s L d\right)+s L 2
\end{gathered}
$$


10 Chunhua Wang \& Xin Zhang \& Yichuang Sun \& Haijun Peng

$$
\begin{aligned}
& Z_{s 1}=s L_{s}+1 / s C_{g s 1 a} \\
& R_{d 1}=r_{d s 1 b}+1 / g_{m 1 b}
\end{aligned}
$$

The voltage gain of the second stage is given by:

$$
\begin{gathered}
A_{2}=\frac{v_{o}}{v_{i n 2}}=\frac{-g_{m 2}+s C_{g d 2}}{\frac{1}{r_{d s 2} / / Z_{d 2}}+s C_{g d 2}} \\
Z_{d 2}=\left(R_{o}+s L_{o}\right) / / Z_{b u f}
\end{gathered}
$$

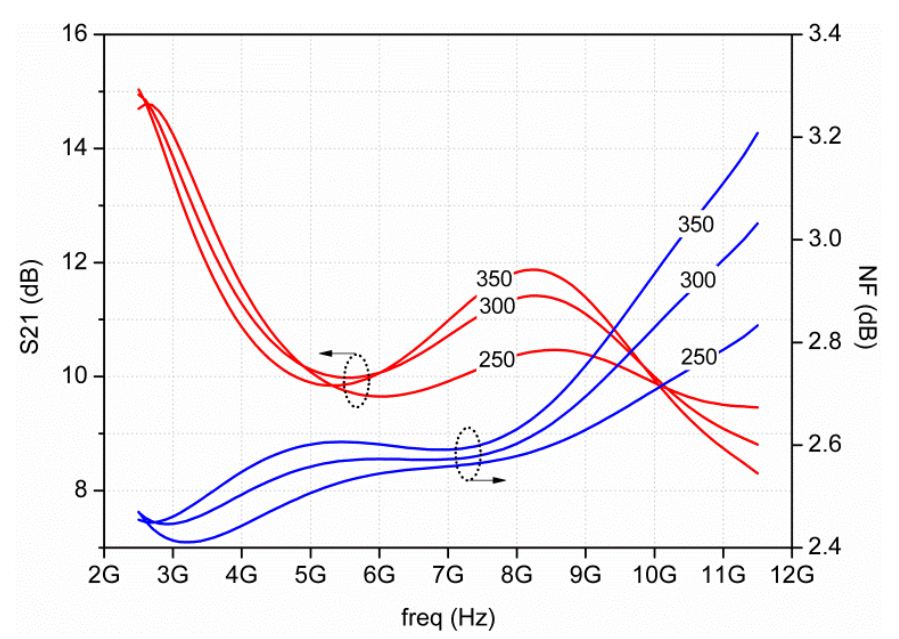

Fig. 5. Simulated gain and NF characteristics with output resistance of $250 \Omega, 300 \Omega$ and $350 \Omega$.

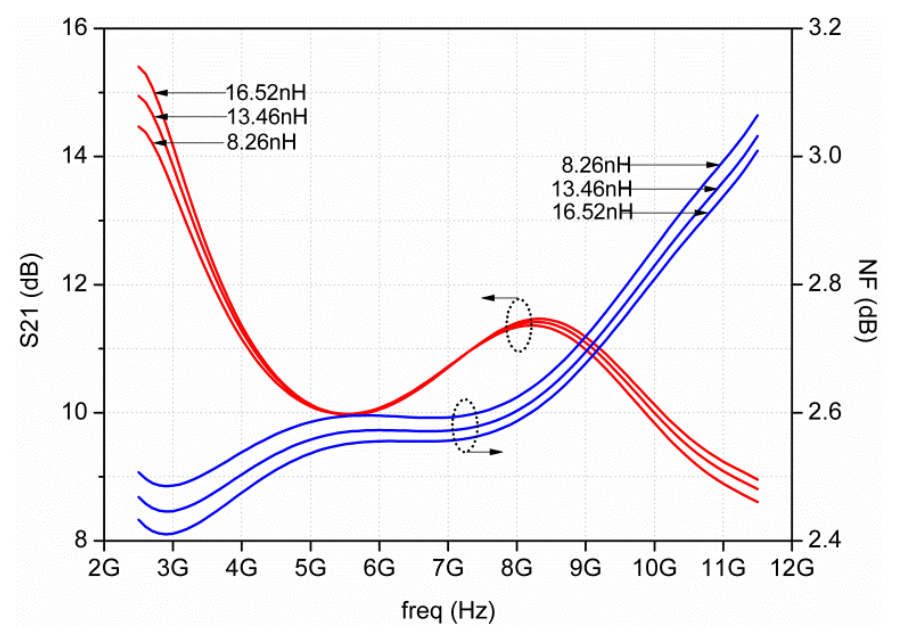

Fig. 6. Simulated gain and NF characteristics with inductance $\mathrm{L}_{\mathrm{o}}$ of $8.26 \mathrm{nH}, 13.46 \mathrm{nH}$ and $16.52 \mathrm{nH}$. 
The gain increases with the increase of $R_{o}$, but too large $R_{o}$ will worsen output matching. Inductor $L_{2}$ improves gain at high frequency resonating with parasitic capacitor at the drain node of M1a. A small capacitor $C_{2}$ improves the gain flatness. The addition of M1b decreases the gain slightly, due to the presence of the small $R_{d 1}$. However, we can increase $g_{m 2}$ and decrease $g_{m l b}$ to compensate for the degradation of gain. Simulated gain and NF characteristics with different output resistances are shown in Fig. 5. In Fig. 5, the variation of transfer characteristics is demonstrated when the values of output resistances $R_{o}$ are equal to $250 \Omega, 300 \Omega$ and $350 \Omega$. The gain and NF variations with resistance variation of $\pm 16.7 \%$ are less than $1 \mathrm{~dB}$ and $0.5 \mathrm{~dB}$, respectively. In addition, inductance $L_{o}$ will affect output matching in the way of extending the bandwidth. Simulation results in Fig. 6 show that the gain variation is less than $0.2 \mathrm{~dB}$. Meanwhile, noise figure degradation is less than $0.5 \mathrm{~dB}$ with inductance variation. In this paper, $R_{o}=300 \Omega$ and $L_{o}$ $=13.46 \mathrm{nH}$.

\subsection{Noise Analysis}

The noise figure of the first stage is critical to the whole circuit, especially when the CG amplifier owns an effective power gain. The channel noise and gate-induced noise of M1b appearing at the LNA output is

$$
\begin{gathered}
\overline{i_{n d, M 1 b}^{2}}=4 k T \frac{\gamma}{\alpha} g_{m 1 b} \\
\overline{i_{n g, M 1 b}^{2}}=4 k T \frac{\delta \alpha \omega^{2} C_{g s 1 b}^{2}}{5} \cdot \frac{g_{m 1 b}}{\left(g_{m 1 b}+g_{m 2}\right)^{2}}
\end{gathered}
$$

The noise contribution from M1b is proportional to its transconductance, which is much smaller than $g_{m l a}$. The noise factor of the proposed linearized cascode CG-LNA can be calculated as follows:

$$
\begin{aligned}
F \approx & +\frac{\gamma}{\alpha} \frac{1}{g_{m 1 a} R_{s}}+\frac{\delta \alpha}{5 g_{m 1 a} R_{s}}\left(\frac{\omega}{\omega_{T}}\right)^{2}+\frac{R_{d 1}}{\left(\omega^{2} L_{d}^{2}+R_{d 1}^{2}\right) R_{s} g_{m 1 a}^{2}\left(\frac{Z_{i n}(s)}{Z_{i n}(s)+R_{s}}\right)^{2}} \\
& +\frac{\gamma}{\alpha} \frac{1}{g_{m 1 a} R_{s}} \cdot \frac{g_{m 1 \mathrm{~b}}}{g_{m 1 a}}+\frac{\delta \alpha}{5 g_{m 1 a} R_{s}}\left(\frac{\omega}{\omega_{T}}\right)^{2} \cdot \frac{g_{m 1 \mathrm{~b}}}{g_{m 1 a}} \cdot \frac{g_{m 1 b}^{2}}{\left(g_{m 1 \mathrm{~b}}+g_{2}\right)^{2}}
\end{aligned}
$$

where $\gamma, \alpha, \delta$ are process dependent parameters, ${ }^{18 .}$ and $Z_{i n}(s)$ is derived in Eq. (22). In Eq. (35), the last two terms are the additional noise contribution from the linearization circuit. The $5^{\text {th }}$ and $6^{\text {th }}$ terms are the channel noise of $\mathrm{M} 1 \mathrm{~b}$, which is smaller than the channel noise of M1a by a factor of $g_{m l b} / g_{m l a}(0.27$ in our design). To properly choose the size of M1a and M1b transistors, the optimum size of M1a and M1b are already set to allow the aforementioned noise and PD to take effect. Adding the auxiliary transistor M1b together with the main transistor M1a allows the equivalent $g_{m}$ to reshape the LNA IIP3 
drastically as can be seen in Fig. 7. Comparing Eq. (17) with Eq. (35), in the PD technique, the value of $g_{m l b} / g_{m l a}$ in IIP3 and noise figure is different from that in $g_{m}$ reduction by Eq. (18). Thus, the degradation in NF is slightly less than $0.4 \mathrm{~dB}$ over the entire bandwidth. The PD technique does not affect the NF appreciably based on the above discussion. In this paper, $g_{m l b}=8.7 \mathrm{mS}$ and $g_{m 2}=12.4 \mathrm{mS}$.

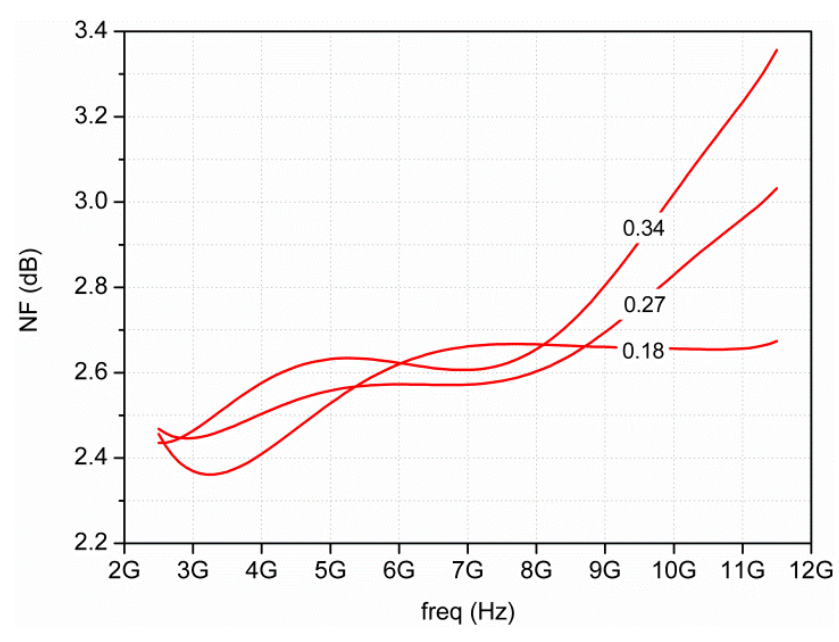

Fig. 7. NF versus $g_{m l b} / g_{m l a}$ of $0.18,0.27$ and 0.34 .

\subsection{Process and Temperature Variations}

To investigate the temperature sensitivity of the PD linearization technique, IIP3 simulations were conducted at three corners including fast process $\left(-25^{\circ} \mathrm{C}\right.$, corner 1$)$, typical process $\left(65^{\circ} \mathrm{C}\right.$, corner 2$)$ and slow process $\left(100^{\circ} \mathrm{C}\right.$, corner 3$)$. In all cases, IIP3 tests are made in $100 \mathrm{MHz}$ tone spacing and $\mathrm{Pin}=-20 \mathrm{dBm}$. The $4.5 \mathrm{GHz}, 8 \mathrm{GHz}$ and $10.5 \mathrm{GHz}$ in Table 1 are treated as the intermodulation frequencies. At the same frequency and the different process corners, IIP3 varies because $g_{m l a}(T)$ and $g_{m l b}(T)$ changes with temperature $T$. At the same process corner and the different frequencies, IIP3 also varies but this is due to the different characteristics and matching of MOSFETs.

Table 1. Simulation results of the proposed UWB LNA for three corners.

\begin{tabular}{lllll}
\hline Linearity & Inter-modulation freq. & Corner 1 & Corner 2 & Corner 3 \\
\hline \multirow{2}{*}{ IIP3 $(\mathrm{dBm})$} & $4.5 \mathrm{GHz}$ & 1.0 & -2.1 & -4.2 \\
& $8 \mathrm{GHz}$ & 6.6 & 6.2 & 4.4 \\
& $10.5 \mathrm{GHz}$ & 2.7 & -1.6 & -3.0 \\
\hline
\end{tabular}




\subsection{Monte Carlo Simulation}

The Monte Carlo simulations were performed to check the stability against the process and temperature variations and the mismatch of MOSFETs. To check the effect of process variation and the mismatch of MOSFETs, pre-layout simulation was conducted with a $\pm 20 \%$ variation in the sizes of M1a, M1b and M2 and for the typical process and $65^{\circ} \mathrm{C}$ corner. All parameters were simulated at $8 \mathrm{GHz}$ with two hundred simulation results.

Figure 8 shows the Monte Carlo simulation for S21. The mean value of $11.17 \mathrm{~dB}$ and the standard deviation of around 0.02 are obtained. So S21 does not vary drastically with the mismatch. The simulation result of IIP3 is presented in Fig. 9, which indicates a center-oriented graph. IIP3 can decrease to $3.5 \mathrm{dBm}$ which is $2.5 \mathrm{~dB}$ below the mean value. Considering that the initial simulation value of IIP3 is $6.2 \mathrm{dBm}$ for the typical process (corner 2), IIP3 tends to be worse with mismatches. Nevertheless, the simulated value of IIP3 is still $5 \mathrm{~dB}$ higher than the largest IIP3 without the linearization method as will be shown later (in Fig. 14).

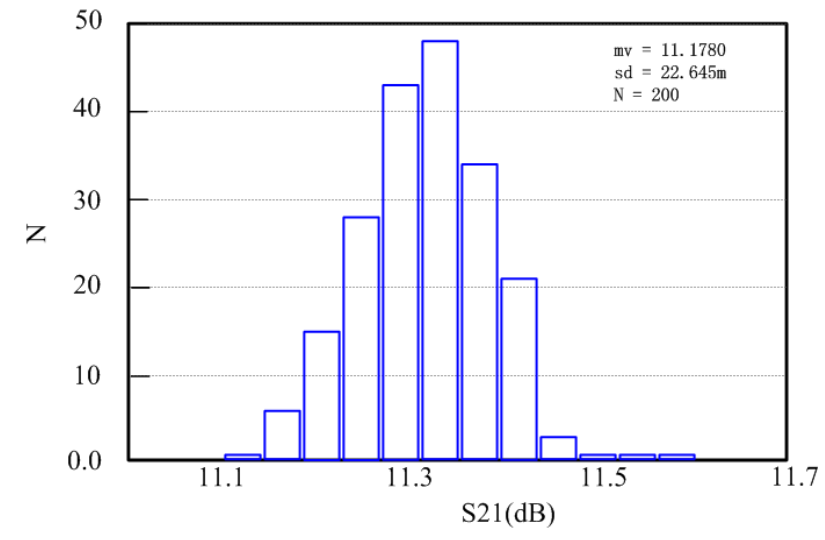

Fig. 8. Monte Carlo simulation results of S21.

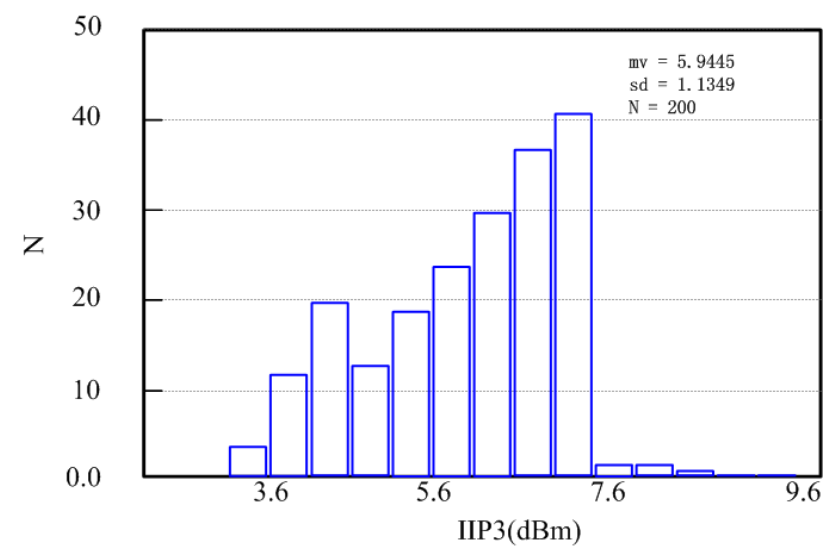

Fig. 9. Monte Carlo simulation results of IIP3. 


\section{Simulation Results and Discussion}

The proposed LNA was simulated using Cadence with CHRT $0.18 \mu \mathrm{m}$ RF process. By employing the folded-cascode topology, the supply voltage of the LNA can be reduced by one transistor overdrive. To satisfy the requirements for wideband matching and lowvoltage operation, the supply voltage suitable for this LNA can reduce to $1 \mathrm{~V}$. However, for similar circuits in Refs. 15. and 19. , a supply voltage higher than $1 \mathrm{~V}$ is needed. In order to reduce the power consumption, the width of M1b was chosen to very small.

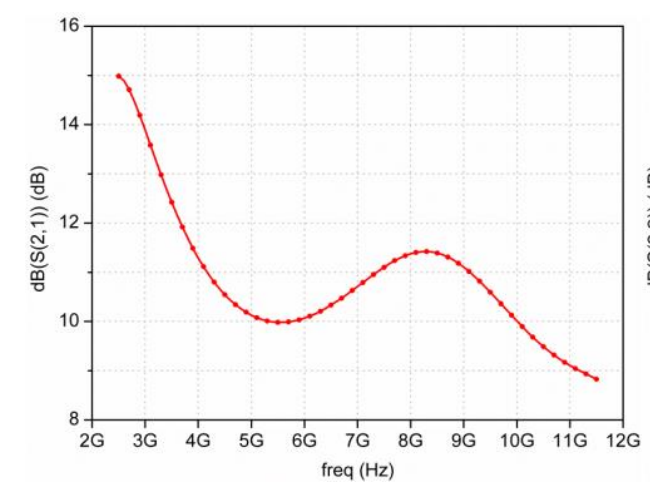

Fig. 10. Power gain (S21).

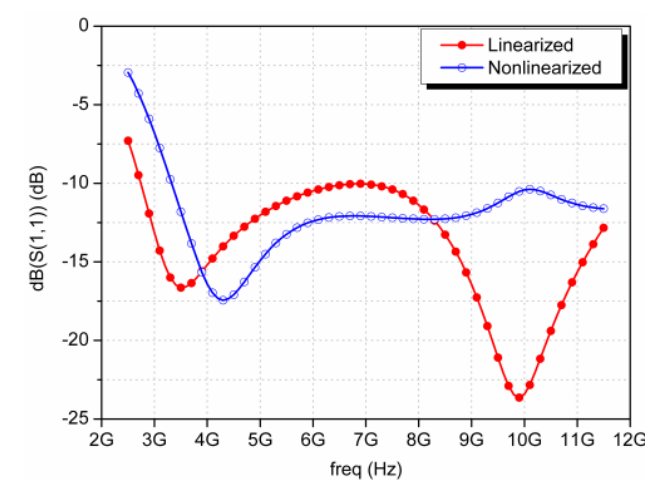

Fig. 12. Input reflection coefficient (S11).

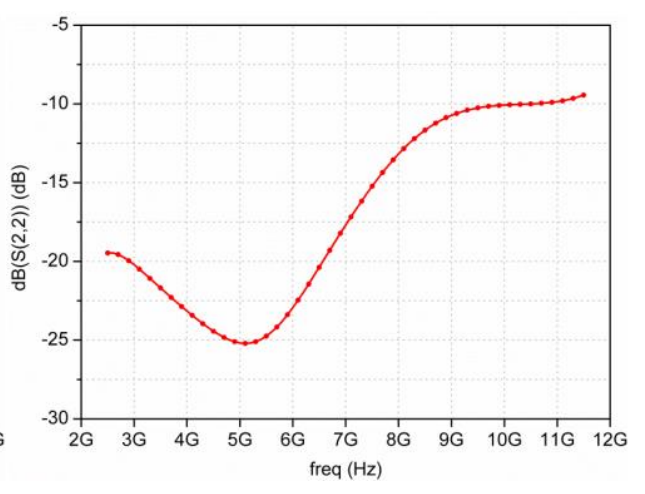

Fig. 11. Output reflection coefficient (S22).

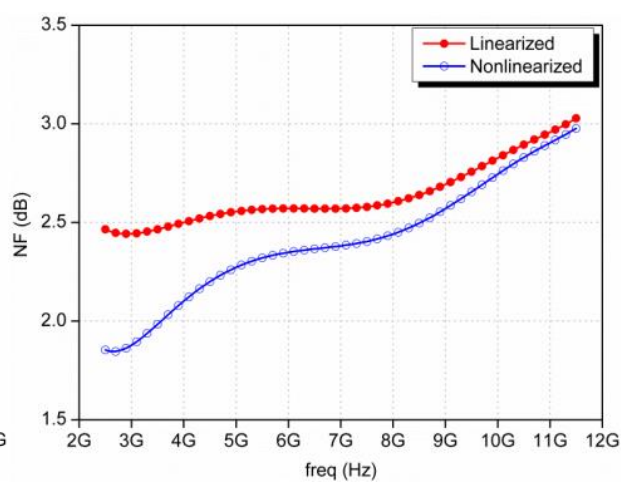

Fig. 13. NF of the proposed LNA.

In the design of the proposed CG-LNA, we chose $\mathrm{Wm} 1 \mathrm{a}=4 * 28 \mathrm{um}, \mathrm{Wm} 1 \mathrm{~b}=20 \mathrm{um}$, $\mathrm{Wm} 2=4 * 16 \mathrm{um}, \mathrm{WmB}=\mathrm{WmF}=4 * 13 \mathrm{um}, \mathrm{C} 1=1.0 \mathrm{pF}, \mathrm{L} 1=1.8 \mathrm{nH}, \mathrm{Ls}=4.8 \mathrm{nH}, \mathrm{Ld}=1.93 \mathrm{nH}$, $\mathrm{Lc}=9.2 \mathrm{nH}, \mathrm{Vb} 1=0.68 \mathrm{~V}, \mathrm{Vb} 2=0.79 \mathrm{~V}$. The maximum power gain is $15.0 \mathrm{~dB}$ at $2.5 \mathrm{GHz}$ as shown in Fig. 10. The output reflection coefficient S22 and input reflection coefficient $\mathrm{S} 11$ are less than $-10.7 \mathrm{~dB}$ over the frequency range from $2.5 \mathrm{GHz}$ to $11.5 \mathrm{GHz}$ which are shown in Fig. 11 and Fig. 12, respectively. The NF is $1.8 \sim 2.9 \mathrm{~dB}$ across the frequency band of interest as shown in Fig. 13. The isolation is less than $-30 \mathrm{~dB}$. The pre-layout simulation indicates that the IIP3 improvement greater than $-2.1 \mathrm{dBm}$ is achieved in the worst case at $4.5 \mathrm{GHz}$, and an IIP3 of $6.2 \mathrm{dBm}$ is obtained at $8 \mathrm{GHz}$ as shown in Table 1 
and Fig. 14. The post-distortion technique can improve the IIP3 by 5 10dB. Figure 15 shows the microphotography of the fabricated circuit with a chip area of $1.0 * 1.1 \mathrm{~mm}^{2}$ including the pads. Operated under a supply voltage of $1 \mathrm{~V}$, the post-layout simulation shows that the LNA consumes a DC power of $5.4 \mathrm{~mW}$ and the IIP3 of $5.6 \mathrm{dBm}$ at $8 \mathrm{GHz}$.

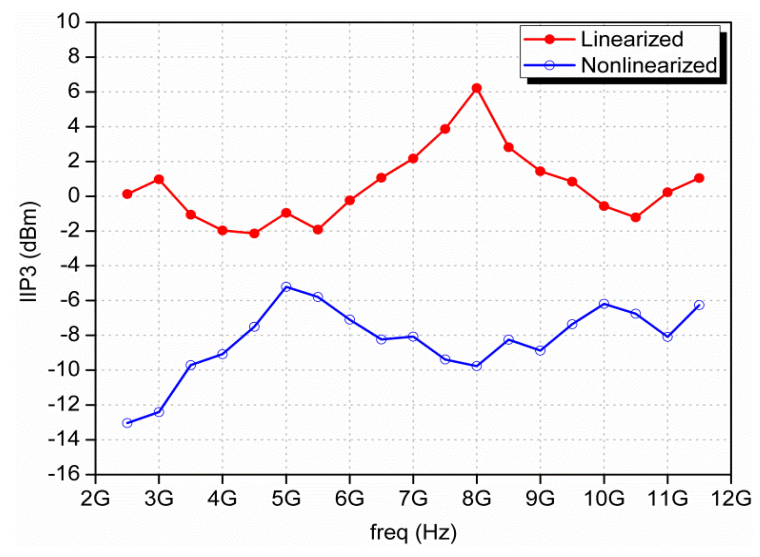

Fig. 14. Simulated IIP3 of CG-LNA with and without linearization, using 100MHz spacing two-tone with -20 dBm power level.

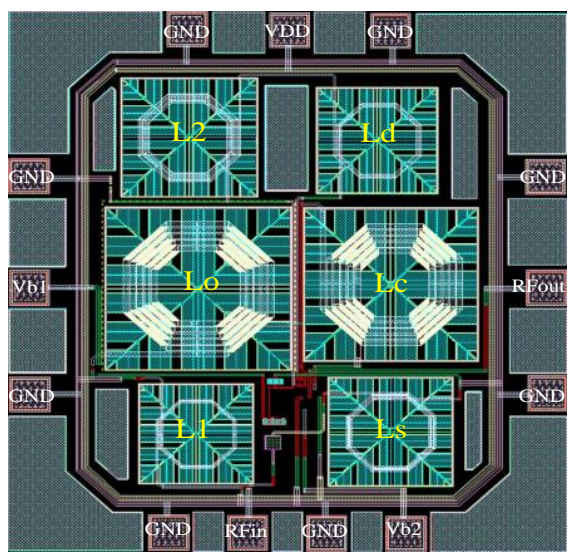

Fig. 15. Microphotograph of the proposed LNA.

Table 2 summaries the performance of the proposed CG-LNA and comparison with the performances of some reported LNAs in the literature. From the data given in the table can be seen that the frequency range of the proposed LNA over $2.5 \mathrm{GHz}-11.5 \mathrm{GHz}$ due to embed a band-pass LC filter in the folded-cascode circuit, and full coverage of the maximum UWB standard. Moreover, the S11 are less than $-10 \mathrm{~dB}$ in the whole working bandwidth, it shows that the LNA possesses good input impedance matching within the entire $9 \mathrm{GHz}$ band. A post-distortion (PD) technique employing an auxiliary transistor is applied in the transconductance stage to improve the linearity, and the input third-order 
intercept point (IIP3) reached $5.6 \mathrm{dBm}$ at $8 \mathrm{GHz}$. The noise figure (NF) is lower than 4.0dB. The proposed UWB LNA achieves comparable IIP3 and its NF is even less than the one in the condition of the best linearity in Refs. 14 and 15.The LNA consumes $5.4 \mathrm{~mW}$ power under a $1 \mathrm{~V}$ supply voltage.

Table 2. Performance Summary and Comparison of the Recently Reported Broadband LNAs.

\begin{tabular}{|c|c|c|c|c|c|c|c|c|c|c|}
\hline Ref & $\begin{array}{l}\text { BW } \\
{[\mathrm{GHz}]}\end{array}$ & $\begin{array}{l}\mathrm{NF} \\
{[\mathrm{dB}]}\end{array}$ & $\begin{array}{l}\text { S11 } \\
{[\mathrm{dB}]}\end{array}$ & $\begin{array}{l}\mathrm{S} 21 \\
{[\max ]}\end{array}$ & $\begin{array}{l}\text { IIP3 } \\
{[\mathrm{dBm}]}\end{array}$ & $\begin{array}{l}\text { Power } \\
{[\mathrm{mW}]}\end{array}$ & $\begin{array}{l}\text { FOM } \\
\text { I }\end{array}$ & $\begin{array}{l}\text { FOM } \\
\text { II }\end{array}$ & $\begin{array}{l}\text { Tech. } \\
\text { [um] }\end{array}$ & Topology \\
\hline [1. ] & $3.1-5.0$ & $4.7-5.0$ & $<-12.7$ & 11.5 & -8.0 & 5.7 & 0.24 & 0.16 & 0.18 & Feedback \\
\hline [10. ] & $0.2-3.8$ & $2.8-3.4$ & $<-9$ & 19.0 & -4.2 & 5.7 & 5.66 & 2.15 & 0.13 & CG-CS \\
\hline [11. ] & $2.3-6.0$ & 4.8 & $<-7$ & 12.7 & 10.6 & 8.3 & 0.95 & 10.9 & 0.13 & Reuse-CS \\
\hline [13. ] & $0.2-5.2$ & 3.5 & -- & 15.6 & 0 & 21 & 1.16 & 1.16 & 0.065 & Balun-CG \\
\hline [14. ] & $7.2-9.1$ & $3.9-4.7$ & $<-9$ & 10.0 & 2.0 & 16 & 0.26 & 0.41 & 0.18 & Cascode \\
\hline [15. ] & $2.5-10.0$ & $2.7-3.3$ & $<-10.2$ & 10.0 & $3.5-9$ & 2.6 & 6.6 & 36.5 & 0.13 & $\begin{array}{l}\text { Folded-CG- } \\
\text { PD }\end{array}$ \\
\hline [19. ] & $3.1-10.6$ & $2.1-2.9$ & $<-9.9$ & 16.5 & -6.0 & 9 & 7.16 & 1.80 & 0.13 & Feedback \\
\hline $\begin{array}{l}\text { This } \\
\text { work }^{\mathrm{a}}\end{array}$ & $2.5-11.5$ & $1.8-2.9$ & $<-10.7$ & 15.0 & 6.2 & 5.3 & 10.9 & 45.4 & 0.18 & $\begin{array}{l}\text { Folded-CG- } \\
\text { PD }\end{array}$ \\
\hline $\begin{array}{l}\text { This } \\
\text { work }^{\text {b }}\end{array}$ & $2.5-11.5$ & $3.2-4.0$ & $<-10.0$ & 12.8 & 5.6 & 5.4 & 5.64 & 20.5 & 0.18 & $\begin{array}{l}\text { Folded-CG- } \\
\text { PD }\end{array}$ \\
\hline
\end{tabular}

a: the pre-layout simulation

b: the post-layout simulation

For the comparison of different topologies, we include two figures of merit (FOMs) in Table 2:

$$
\begin{gathered}
\text { FOM }-I=\frac{\operatorname{Gain}_{\text {avv }}[\mathrm{abs}] \times B W[\mathrm{GHz}]}{P_{d c}[\mathrm{~mW}] \times\left(F_{\text {ave }}-1\right)} \\
F O M_{-} I I=\frac{I I P 3_{\text {ave }}[\mathrm{mW}] \times \text { Gaiin }_{\text {ave }}[\mathrm{abs}] \times B W[\mathrm{GHz}]}{P_{d c}[\mathrm{~mW}] \times\left(F_{\text {ave }}-1\right)}
\end{gathered}
$$

where Gain ave $_{\text {is }}$ the average gain, $F_{\text {ave }}$ is the average noise factor over the frequency range, and $P_{d c}$ is the power consumption of the LNA core.

From Eq. (36) and Eq. (37), the FOM_I and FOM_II are synthetically evaluating coefficient about LNA, the higher the value, the better the combination property. $F O M_{-} I{ }^{20}{ }^{20}$ which does not include linearity, and $F O M_{-} I I,{ }^{21}$. which includes linearity,

From Table 2, our LNA also exhibits comparable FOM_I, and has much better FOM_II when compared to the other UWB LNAs. Therefore, through the comparison table, proved the proposed UWB LNA has excellent comprehensive performance even under an extremely low supply voltage. 
Finally, we consider the stability of the proposed LNA circuit. The necessary and sufficient condition of amplifier is:

$$
\left\{\begin{array}{l}
k_{0}>1 \\
|\Delta|<1
\end{array}\right.
$$

where $k_{0}$ is stability discriminant factor, $\Delta$ and $k_{0}$ can be calculated by:

$$
\begin{aligned}
& \Delta=S 11 \cdot S 22-S 21 \cdot S 12 \\
& k_{0}=\frac{1-|S 11|^{2}-|S 11|^{2}+|\Delta|^{2}}{2|S 12||S 21|}
\end{aligned}
$$

The corresponding parameters of our proposed novel CG-LNA is calculated, it can achieve stability condition.

\section{Conclusions}

In this paper, a low-voltage highly linear CMOS LNA has been proposed. The postlayout simulation results show that the designed LNA achieves a $5.6 \mathrm{dBm}$ IIP3 peak, a maximum gain of $12.8 \mathrm{~dB}$, and consumes $5.4 \mathrm{~mW}$ power, under a $1 \mathrm{~V}$ supply voltage. The proposed post-linearization technique improves the IIP3 by $5 \sim 10 \mathrm{~dB}$. The proposed LNA can be used in low-voltage wideband receivers for multi-standard systems such as UWB, Bluetooth, WLAN, ZigBee, WiMAX, etc. It is particularly suitable for high-speed mobile applications because of its high linearity.

\section{Acknowledgments}

The authors would like to thank the editors and anonymous reviewers for their valuable comments which have helped us improve the paper. This research was supported by the National Natural Science Foundation of China (No. 61571185, 61674054), the Natural Science Foundation of Hunan Province, China (No. 2016JJ2030) and the Open Fund Project of Key Laboratory of Hunan Universities (No. 15K027).

\section{References}

1. Z.Y. Huang, C. C. Huang, C. C. Chen, C. C. Hung, and C. F. Jou, A CMOS low-noise amplifier with impedance feedback for ultra-wideband wireless receiver system, IEEE International Symposium on VLSI Design, Automation and Test (2008), pp. 51-54.

2. L. Pantoli, Transient-based conversion matrix approach for nonlinear stability analysis, Electronics Letters 50 (2014) 923-925.

3. H. Zhang and E. Sanchez-Sinencio, Linearization techniques for CMOS low noise amplifiers: a tutorial, IEEE Trans. Circuits Syst. I. Regul 58 (2011) 22-36.

4. R. I. Saadi and A. Hakimi, Low-power and low-noise complementary metal oxide semiconductor distributed amplifier using the gain-peaking technique, International Journal of Circuit Theory and Applications (2016) DOI: 10.1002/cta.2226. 
18 Chunhua Wang \& Xin Zhang \& Yichuang Sun \& Haijun Peng

5. L. Zhao and C. Wang, A Low Power High Gain CMOS LNA with Multiple-Feedback Network for Low Voltage UWB Receiver, Journal of Circuits System and Computers 25 (2016) 1650051.

6. A. F. Ponchet, E. M. Bastida, C. A. Finardi, R. R. Panepucci, S. Tenenbaum, S. Finco and J. W. Swart, A design methodology for low-noise CMOS transimpedance amplifiers based on shunt-shunt feedback topology, In 29th Symposium on Integrated Circuits and Systems Design (SBCCI) (2016), pp. 1-6.

7. W. H. Chen, G. Liu, B. Zdravko and A. M. Niknejad, A highly linear broadband CMOS LNA employing noise and distortion cancellation, IEEE Journal of Solid-State Circuits 43 (2008) 1164-1176.

8. V. Aparin and L. E. Larson, Modified derivative superposition method for linearizing FET low-noise amplifiers, IEEE Trans. Microwave Theory and Techniques 53 (2005) 571-581.

9. Y. Shim, C. Kim, J. Lee, S. G. Lee, Design of full band UWB common-gate LNA, IEEE Microwave and Wireless Components Letters 17 (2007) 721-723.

10. H. Wang, L. Zhang and Z. Yu, A wideband inductorless LNA with local feedback and noise cancelling for low-power low-voltage applications, IEEE Trans. Circuits Syst. I. Regul 57 (2010) 1993-2005.

11. K. Choi, T. Mukherjee and J. Paramesh, A linearity-enhanced wideband low-noise amplifier, IEEE Radio Frequency Integrated Circuits Symposium 54 (2010) 127-130.

12. A. Mabrouki, T. Taris, Y. Deval and J. Begueret, CMOS low-noise amplifier linearization through body biasing, 2009 IEEE International Symposium on Radio-Frequency Integration Technology (RFIT) (2010), pp. 150-153.

13. S. C. Blaakmeer, E. A. M. Klumperink, D. M. W. Leenaerts and B. Nauta, Wideband balunLNA with simultaneous output balancing, noise-canceling and distortion-canceling, IEEE Journal of Solid-State Circuits 43 (2008) 1341-1350.

14. C. L. Yang, W. L. Hsieh and Y. C. Chiang, A fully integrated and high linearity UWB LNA implemented with current-reused technique and using single-biasing voltage, European Conference on Wireless Technologies (2007), pp. 94-97.

15. H. Zhang, X. Fan and E. S. Sinencio, A low-power, linearized, ultra-wideband LNA design technique, IEEE Journal of Solid-State Circuits 44 (2009) 320-330.

16. T. W. Kim, A common gate mixer with transconductance nonlinearity cancellation, IEEE 8th International Conference on ASIC (2009), pp. 458-460.

17. B. Leung, VLSI for Wireless Communication (Prentice Hall: Englewood Cliffs, 2002), p. 147.

18. T. Lee, The Design of CMOS Radio-frequency Integrated Circuits (Cambridge Univ. Press, 1998), p. 164.

19. M. T. Reiha and J. R. Long, A $1.2 \mathrm{~V}$ reactive-feedback $3.1-10.6 \mathrm{GHz}$ low-noise amplifier in 0.13 um CMOS, IEEE Journal of Solid-State Circuits 42 (2007) 1023-1033.

20. S. Shekhar, J. S. Walling and D. J. Allstot, Bandwidth extension technique for CMOS amplifiers, IEEE Journal of Solid-State Circuits 41 (2006) 2424-2438.

21. A. Amer, E. Hegazi and H. Ragai, A low-power wideband CMOS LNA for WiMax, IEEE trans. Circuits Syst. II, Express Briefs 54 (2007) 4-8. 